\title{
Dark Energy and Modified Gravity
}

\section{Philippe Brax*}

Institut de Physique Théorique, CEA, IPhT, CNRS, URA 2306, F-91191 Gif/Yvette Cedex, France

E-mail: philippe.braxdeea.fr

The acceleration of the expansion of the Universe may be due to either a new matter component, dark energy, or a modification of gravity on large scales. In fact, modifying gravity always involves a scalar degree of freedom which can lead to dark energy. Locally, in the solar system, gravity is extremely well described by General Relativity. Hence any modification of gravity must have screened effects in our local environment. We review the known screening mechanisms: chameleon, K-mouflage and Vainshtein. For the chameleon mechanism as exemplified by $f(R)$ models, the local tests imply that the effective equation of state of dark energy is very close to -1 in the late time Universe. For Galileons with the Vainshtein mechanism, self-acceleration is possible with a varying equation of state. In both cases, modifications of gravity are accompanied by a change in the growth of structure.

Frontiers of Fundamental Physics 14 - FFP14,

15-18 July 2014

Aix Marseille University (AMU) Saint-Charles Campus, Marseille

\footnotetext{
* Speaker.
} 


\section{Introduction}

Since the discovery of the acceleration of the expansion of the Universe [1], dark energy [2] and modified gravity [3] have been studied as a possible theoretical framework for the description of the late time Universe. In the last few years, it has become clearer and clearer that dark energy and modified gravity mostly involve the physics of very light scalar fields [4]. In dark energy models, this is by-product of the requirement that the equation of state must be close to -1 . For modified gravity, massive gravitons always involve a scalar polarisation out of their 5 helicity states. However, the existence of light scalar fields is extremely constrained by local solar system experiments on fifth forces (Cassini) [5] or the strong equivalence principle (Lunar ranging) [6]. In this environment, the scalar field must be screened and see its effects on matter largely reduced. This is feasible is three different ways: chameleon [7-9], K-mouflage [10] and Vainshtein [11]. In this paper, we will review how these mechanisms operate and we will give typical examples of models realising the chameleon and Vainshtein properties. For the chameleons, the $f(R)$ theories in the large curvature limit [12] allow one to envisage models where large scalar structure are altered whereas the background cosmology is essentially the one of $\Lambda$-CDM. On the other hand Galileons [13], and herein the cubic ones, are amenable to self-acceleration with a varying equation of state and also modifications of the growth of structure.

\section{Screening Mechanisms}

\subsection{Background effects}

Screening mechanisms $[4,14]$ can be described by considering theories with second order equations of motion only. In a given environment where the scalar field takes a background value $\varphi_{0}(t)$ and expanding to second order the Lagrangian of the fluctuations compared to the background $\delta \varphi=\varphi-\varphi_{0}(t)$, we obtain

$$
\mathscr{L}=-\frac{Z\left(\varphi_{0}\right)}{2}(\partial \delta \varphi)^{2}-\frac{m^{2}\left(\varphi_{0}\right)}{2}(\delta \varphi)^{2}-\beta\left(\varphi_{0}\right) \frac{\delta \varphi}{M_{\mathrm{Pl}}} \delta \rho_{m}
$$

where $\delta \rho_{m}$ is the change of the matter density compared to the background value. Test particles follow the geodesics of the total potential $\Psi=\Psi_{\mathrm{N}}+\beta\left(\varphi_{0}\right) \frac{\delta \varphi}{M_{\mathrm{Pl}}}$, where the Newtonian potential $\Psi_{\mathrm{N}}$ satisfies the Poisson equation $\nabla^{2} \Psi_{\mathrm{N}}=4 \pi A\left(\varphi_{0}\right) \mathscr{G}_{\mathrm{N}} \delta \rho_{\mathrm{m}}$ and $\delta \varphi$ is due to the presence of matter overdensities. Big Bang Nucleosynthesis imposes that the overall variation of particles masses is less than ten percent since $\mathrm{BBN}$, i.e $\left|\frac{\Delta A}{A}\right| \leq 0.1$ where $\Delta A$ is the variation of A. implying that $A \simeq 1$. Screening corresponds to the reduction of the effect of the scalar field from the linear case of a point particle coupled with a strength $\beta\left(\varphi_{0}\right)$ to matter, i.e. $\Psi$ is smaller than $\left(1+2 \beta^{2}\left(\varphi_{0}\right)\right) \Psi_{\mathrm{N}}$.

\subsection{Chameleon}

For canonically normalised scalars and at the linear level [9], the equations of motion give that

$$
\Psi=\left[1+\frac{2 \beta^{2}\left(\varphi_{0}\right)}{1+m^{2}\left(\varphi_{0}\right) a^{2} / k^{2}}\right] \Psi_{\mathrm{N}}
$$


where $k$ is the comoving wave number of interest. General Relativity is recovered on very large scales outside the Compton wavelength, $k / a \lesssim m\left(\varphi_{0}\right)$, and changes to gravity occur inside the Compton wavelength, $k / a \gtrsim m\left(\varphi_{0}\right)$, with a strength $\left(1+2 \beta^{2}\left(\varphi_{0}\right)\right)$. When overdensities become greater, the linear approximation is not valid anymore and screening occurs. The condition for the onset of nonlinear screening, $|\delta \varphi| \sim\left|\varphi_{0}\right|$, can also be written

$$
\left|2 \beta\left(\varphi_{0}\right) \Psi_{\mathrm{N}}\right| \gtrsim\left|\frac{\varphi_{0}}{M_{\mathrm{Pl}}}\right| .
$$

Viable $f(R)$ models are chameleons which we will present in the following section.

\subsection{K-mouflage and Vainshtein mechanisms}

At the linear level for models dominated by their kinetic term we have that

$$
\Psi=\left[1+\frac{2 \beta^{2}\left(\varphi_{0}\right)}{Z\left(\varphi_{0}\right)}\right] \Psi_{\mathrm{N}}
$$

and screening occurs when

$$
Z\left(\varphi_{0}\right) \gtrsim 1
$$

Non-linearly, the modification of gravity around an overdensity is still suppressed when $Z$ is large. We can expand to leading order

$$
Z(\varphi)=1+a(\varphi) \frac{(\partial \varphi)^{2}}{\mathscr{M}^{4}}+b(\varphi) L^{2} \frac{\square \varphi}{M_{\mathrm{Pl}}}+\ldots,
$$

where $\mathscr{M}$ is a suppression scale characterising the model, $L$ a typical length scale, $a(\varphi)$ and $b(\varphi)$ two functions of order one. Cubic and higher order derivatives are forbidden as they would induce equations of motions of order larger than two.

\subsubsection{Vainshtein scenario}

When $a=0$, the suppression of the scalar field effect is due to the Vainshtein effect when $\frac{\left|\nabla^{2} \varphi\right|}{M_{\mathrm{Pl}}} \gtrsim L^{-2}$, implying that screening occurs when

$$
\left|\nabla^{2} \Psi_{\mathrm{N}}\right| \gtrsim \frac{\left|\nabla^{2} \varphi\right|}{2 \beta M_{\mathrm{Pl}}} \gtrsim \frac{1}{2 \beta L^{2}}
$$

when $\beta$ is a slowly varying function of $\varphi$. Therefore the screening criterion depends on the spatial curvature which must be large enough.

Taking the Newtonian potential around a dense object of mass $m$, screening occurs inside the Vainshtein radius

$$
R_{V}=\left(\frac{3 \beta L^{2} m}{4 \pi M_{\mathrm{Pl}}^{2}}\right)^{1 / 3}
$$

For quasi-linear cosmological structures, with a density constrast of $\delta$, screening occurs when

$$
3 A\left(\varphi_{0}\right) \Omega_{\mathrm{m} 0} H_{0}^{2} \delta \gtrsim \frac{1}{\beta\left(\varphi_{0}\right) L^{2}} .
$$


When the scale $L$ is the size of the current Universe $H_{0}^{-1}$, screening occurs when

$$
\delta \gtrsim \frac{1}{3 \Omega_{\mathrm{m} 0} A\left(\varphi_{0}\right) \beta\left(\varphi_{0}\right)},
$$

which is realised for all overdensities with a density contrast larger than a number of order one Hence all quasi-linear structures in the Universe are screened in the Vainshtein models.

\subsubsection{K-mouflage scenario}

When $b=0$, the suppression of the scalar field effect is due to the K-mouflage effect when $|\nabla \varphi| \gtrsim \mathscr{M}^{2}$, implying that a necessary condition for screening is

$$
\left|\nabla \Psi_{\mathrm{N}}\right| \gtrsim \frac{|\nabla \varphi|}{2 \beta M_{\mathrm{Pl}}} \gtrsim \frac{\mathscr{M}^{2}}{2 \beta M_{\mathrm{pl}}}
$$

when $\beta$ is slowly varying function of $\varphi$. Therefore we now have a criterion on the gradient of the gravitational potential, that is, the gravitational acceleration.

Taking the Newtonian potential around a dense object of mass $m$, we find that screening occurs inside the K-mouflage radius

$$
R_{K}=\left(\frac{\beta m}{4 \pi M_{\mathrm{P}} \mathscr{M}^{2}}\right)^{1 / 2}
$$

For quasi-linear cosmological structures, we find that screening occurs when the wave number $k$ characterising a given structure satisfies

$$
k \lesssim 3 \Omega_{\mathrm{m} 0} A\left(\varphi_{0}\right) \beta\left(\varphi_{0}\right) \frac{H_{0}^{2} M_{\mathrm{Pl}}}{\mathscr{M}^{2}} \delta
$$

Taking $\mathscr{M}^{4} \sim 3 \Omega_{\Lambda 0} M_{\mathrm{Pl}}^{2} H_{0}^{2}$ to recover the acceleration of the Universe now, we have that

$$
\frac{k}{H_{0}} \lesssim \sqrt{\frac{3}{\Omega_{\Lambda 0}}} \Omega_{\mathrm{m} 0} A\left(\varphi_{0}\right) \beta\left(\varphi_{0}\right) \delta,
$$

which corresponds to super-horizon scales if $\delta \sim 1$. As a result, quasi-linear objects in the Universe are not screened in K-mouflage models.

\section{Chameleon Modified Gravity}

\subsection{Tomographic theories}

In screened objects of the chameleon type, the mass is so large or the coupling so small that the scalar field is essentially constant. Denoting by $\phi_{c}$ the value inside the object and by $\phi_{\infty}$ the value outside and far away from the body, an approximate solution of the Klein-Gordon equation in the spherical case which describes accurately the outside solution in the screened case [15], is simply

$$
\phi(r)=\phi_{\infty}-2 Q m_{\mathrm{Pl}} \frac{G_{N} M}{r}
$$

where $M$ is the mass of the dense object, $R$ its radius and the scalar charge

$$
Q=\frac{\phi_{\infty}-\phi_{c}}{2 m_{\mathrm{Pl}} \Phi_{N}}
$$


where $\Phi_{N}$ is the value of Newton's potential at the surface of the body $\Phi_{N}=\frac{G_{N} M}{R}$. The scalar charge depends on the environment via $\phi_{\infty}$ and on the properties of the body via $\phi_{c}$ and $\Phi_{N}$. Comparing to the linear solution for a point-like source, the screening criterion [7] for chameleon theories becomes

$$
Q \lesssim \beta_{\infty}
$$

which requires that the scalar charge of a screened object should be smaller than the coupling to matter far away from the object.

These models can be described by scalar-tensor theories defined by the Lagrangian

$$
S=\int d^{4} x \sqrt{-g}\left(\frac{R}{16 \pi G_{N}}-\frac{(\partial \phi)^{2}}{2}-V(\phi)\right)+S_{m}\left(\psi, A^{2}(\phi) g_{\mu v}\right)
$$

where $A(\phi)$ is an arbitrary function. The coupling to matter is simply given by $\beta(\phi)=m_{\mathrm{Pl}} \frac{d \ln A(\phi)}{d \phi}$. The most important feature of these models is that the scalar field dynamics are determined by an effective potential which takes into account the presence of the conserved matter density $\rho$ of the environment

$$
V_{\text {eff }}(\phi)=V(\phi)+(A(\phi)-1) \rho .
$$

Scalar-tensor theories whose effective potential $V_{\text {eff }}(\phi)$ admits a density dependent minimum $\phi(\rho)$ can all be reconstructed tomographically from the sole knowledge of the mass function $m(\rho)$ and the coupling $\beta(\rho)$ at the minimum of the potential $[15,16]$

$$
\frac{\phi(\rho)-\phi_{c}}{m_{\mathrm{Pl}}}=\frac{1}{m_{\mathrm{Pl}}^{2}} \int_{\rho}^{\rho_{c}} d \rho \frac{\beta(\rho) A(\rho)}{m^{2}(\rho)},
$$

where we have identified the mass as the second derivative $m^{2}(\rho)=\left.\frac{d^{2} V_{\text {eff }}}{d \phi^{2}}\right|_{\phi=\phi(\rho)}$ and the coupling $\beta(\rho)=\left.\frac{d \ln A}{d \phi}\right|_{\phi=\phi(\rho)}$. It is simpler to characterise the functions $m(\rho)$ and $\beta(\rho)$ using the time evolution of the matter density of the Universe $\rho(a)=\frac{\rho_{0}}{a^{3}}$ where $a$ is the scale factor whose value now is $a_{0}=1$. This allows one to describe characteristic models in a simple way.

A large class of interesting models with a screening mechanism of the chameleon type consists of the large curvature $f(R)$ models [12] with the action

$$
S=\int d^{4} x \sqrt{-g} \frac{f(R)}{16 \pi G_{N}}
$$

where the function $f(R)$ is expanded in the large curvature regime

$$
f(R)=\Lambda+R-\frac{f_{R_{0}}}{n} \frac{R_{0}^{n+1}}{R^{n}}
$$

where $\Lambda$ is a cosmological constant term and $R_{0}$ is the present day curvature. These models can be reconstructed using the constant $\beta(a)=1 / \sqrt{6}$ and the mass function as a function of the scale factor $a \leq 1$

$$
m(a)=m_{0}\left(\frac{4 \Omega_{\Lambda 0}+\Omega_{m 0} a^{-3}}{4 \Omega_{\Lambda 0}+\Omega_{m 0}}\right)^{(n+2) / 2}
$$

where the mass on large cosmological scale is given by $m_{0}=H_{0} \sqrt{\frac{4 \Omega_{\Lambda 0}+\Omega_{m 0}}{(n+1) f_{0}}}, \Omega_{\Lambda 0} \approx 0.73, \Omega_{m 0} \approx$ 0.27 are the dark energy and matter density fractions now [15]. Apart from for very small redshifts, the dependence of $m(\rho)$ on $a$ is a power law with an exponent $-3(n+2) / 2$. 


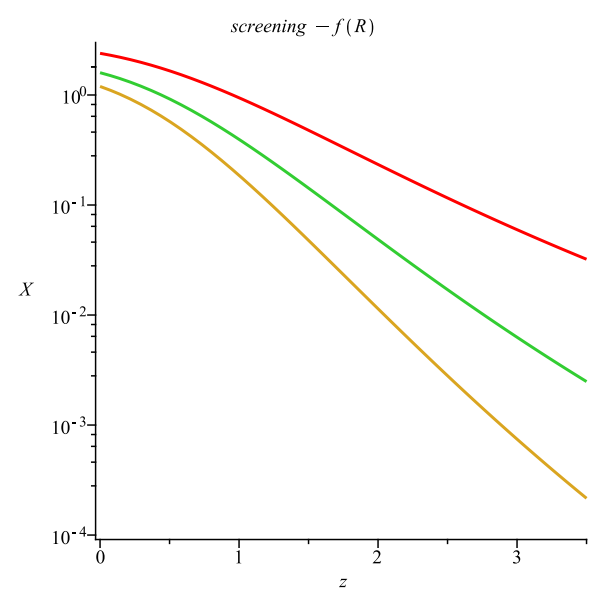

Figure 1: $X(z)$ is represented here for $f(R)$ models with $n=1,2,3$ from top to bottom for astrophysical objects of the galactic type. Local gravitational tests imply that $H_{0} / m_{0} \lesssim 10^{-3}$ for $f(R)$ models, implying that unscreened objects must have a Newtonian potential less than $10^{-6}$ at low redshift and smaller at higher redshift.

The tomographic map allows one to refine the screening criterion as

$$
\frac{1}{m_{\mathrm{Pl}}^{2}} \int_{\rho_{\infty}}^{\rho_{c}} d \rho \frac{\beta(\rho)}{m^{2}(\rho)} \lesssim 2 \beta_{\infty} \Phi_{N}
$$

where $\rho_{\infty}$ is the density far away from the object. The necessary condition for astrophysical objects to be unscreened is that their Newtonian potential satisfies

$$
\Phi_{N} \lesssim \frac{H_{0}^{2}}{m_{0}^{2}} X(z)
$$

where we have introduced the function

$$
X(z)=\frac{9}{2 f(a(z))} \int_{a_{c}}^{(z+1)^{-1}} \frac{f(a) \Omega_{m}(a) H^{2}(a)}{a g(a)} d a
$$

and we have defined the dimensionless functions $m^{2}(a)=m_{0}^{2} g(a)$ and $\beta(a)=\beta_{0} f(a)$. For the Milky Way we have $a_{c} \equiv a_{G} \sim 10^{-2}$. We have plotted the variation of $X(z)$ as a function of the redshift $z$ for $f(R)$ models in Fig.1. For low redshift objects, unscreened objects are characterised by $\Phi_{N} \lesssim \frac{H_{0}^{2}}{m_{0}^{2}}$. As the Milky Way, which must be screened to avoid too much disruption in the dynamics of satellite galaxies, is such that $\Phi_{G} \sim 10^{-6}$, the mass of the scalar field in the cosmological background now $m_{0}$ must then satisfy $[?, 16]$

$$
\frac{m_{0}}{H_{0}} \geq\left(\frac{3 \Omega_{m 0}}{2(n+1) \Phi_{G}}\right)^{1 / 2} \gtrsim 10^{3} .
$$

for $f(R)$ models. This implies that the cosmological range of the scalar field must be less than a few Mpc's now. Unscreened astrophysical objects must necessarily have a low Newtonian potential $\Phi_{N} \lesssim 10^{-6}$ when they are at low redshift, and even smaller when their redshift is $z \gtrsim 1$ as $X(z)$ drops below a few percent for $f(R)$ models. 
Local tests of the strong equivalence principle in the solar system carried out by the Lunar Ranging experiment [6] imply that [7] $Q_{\oplus} \leq 10^{-7}$ and the Newtonian potential on earth is $\Phi_{\oplus} \sim$ $10^{-9}$. This implies that

$$
\frac{9}{2} \int_{a_{\oplus}}^{a_{G}} \frac{\beta(a) \Omega_{m}(a) H^{2}(a)}{a m^{2}(a)} d a=Q_{\oplus} \Phi_{\oplus} \lesssim 10^{-16} .
$$

and for large curvature $f(R)$ models, we get that

$$
\frac{m_{0}}{H_{0}} \geq \sqrt{\frac{3 \beta_{0} \Omega_{m 0}}{2(n+1)}} 10^{5-3 n} .
$$

This is a weaker condition than the screening of the Milky Way when $n \gtrsim 1$ and corresponds to $f_{R 0} \lesssim 10^{-4}$ for $n=1$.

For such models, the equation of state at late time is constrained to be [15]

$$
w_{\phi}+1 \sim 3 \beta_{0}^{2} \Omega_{m 0} \frac{\Omega_{m 0}}{\Omega_{\Lambda}} \frac{H_{0}^{2}}{m_{0}^{2}}
$$

which is the one of $\Lambda$-CDM at the $10^{-6}$ level due to the screening of the Milky Way. This implies that such models are essentially equivalent to $\Lambda-C D M$ at the background level at late time and acceleration is due to an effective cosmological constant in the potential $V(\phi)$. This is not the case for the growth of structure where the density contrast $\delta$ satisfies the growth equation in conformal time where $\mathscr{H}=\frac{\dot{a}}{a}$

$$
\ddot{\delta}+\mathscr{H} \dot{\delta}-\frac{3}{2} \Omega_{m} \mathscr{H}^{2}(1+\varepsilon(k, t)) \delta=0
$$

where $\varepsilon(k, t)=\frac{2 \beta^{2}(a)}{1+\frac{m^{2}(a) a^{2}}{k^{2}}}$. The growth of structure in the linear regime is modified from the $\delta \sim a$ behaviour in the matter dominated era to a $k$ - dependent evolution. On large scale outside the Compton wavelength $k \gtrsim a m(a)$ the growth is similar to the one for $\Lambda$-CDM. On the other hand, inside the Compton scale $k \lesssim a m(a)$ growth is modified due to a rescaling of Newton's constant by a factor of $1+2 \beta^{2}(a)$. For $f(R)$ models in the large curvature regime, this enhacement by a factor of $4 / 3$ could be detectable by future surveys such as EUCLID provided the cosmological range of the scalar field $m_{0}^{-1}$ is not well below $1 \mathrm{Mpc}$ where non-linear and baryonic effects could take over modified gravity. With current bounds on $f_{R 0} \lesssim 10^{-7}$ [17] coming from the astrophysics of stars, the eventual detection of these effects on the growth of structure seems marginal.

\section{Galileons}

Galileons are scalar field theories which have equations of motion that are at most second order in derivatives [13]. They are interesting dark energy candidates where an explicit cosmological constant is not compulsory. The Lagrangian in the cubic case reads

$$
\mathscr{L}=-\frac{c_{2}}{2}(\partial \phi)^{2}-\frac{c_{3}}{\Lambda^{3}} \square \phi(\partial \phi)^{2} .
$$

and depends on two constants $c_{2}$ and $c_{3}$. The common scale $\Lambda^{3}=H_{0}^{2} m_{\mathrm{Pl}}$ is chosen to reproduce the acceleration of the late Universe. In a Friedmann-Robertson-Walker background, the equations 


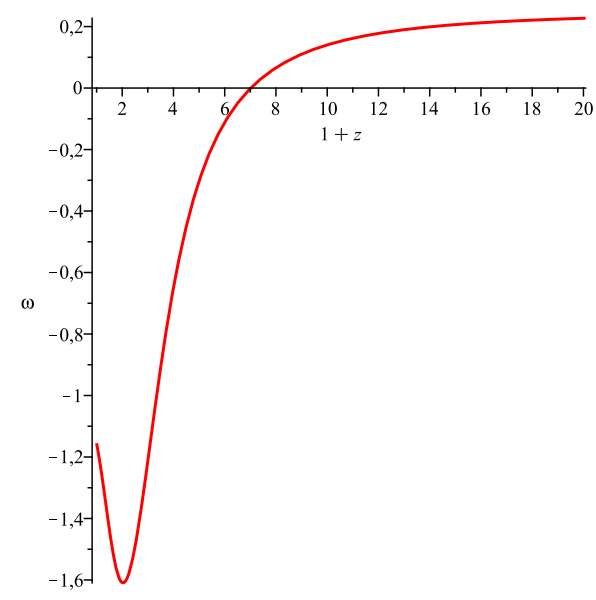

Figure 2: The equation of state $w(z)$ for the cubic Galileon model with $\bar{c}_{2}=-4.3$.

of motion can be simplified using $x=\phi^{\prime} / m_{\mathrm{Pl}}$ where a prime denotes ${ }^{\prime}=d / d \ln a=-d / d \ln (1+z)$ where $a$ is the scale factor and $z$ the redshift. Defining $\bar{x}=x / x_{0}$ and $\bar{H}=H / H_{0}$ where $H$ is the Hubble rate, and the rescaled couplings $\bar{c}_{i}=c_{i} x_{0}^{i}, i=2,3$, where $x_{0}$ is the value of $x$ now, the cosmological evolution satisfies [18]

$$
\bar{x}^{\prime}=-\bar{x}+\frac{\alpha \lambda-\sigma \gamma}{\sigma \beta-\alpha \omega}, \bar{H}^{\prime}=-\frac{\lambda}{\sigma}+\frac{\omega}{\sigma}\left(\frac{\sigma \gamma-\alpha \lambda}{\sigma \beta-\alpha \omega}\right)
$$

where we have introduced

$$
\begin{aligned}
& \alpha=-3 \bar{c}_{3} \bar{H}^{3} \bar{x}^{2}+\frac{\bar{c}_{2} \bar{H} \bar{x}}{6} \\
& \beta=-2 \bar{c}_{3} \bar{H}^{4} \bar{x}+\frac{\bar{c}_{2} \bar{H}^{2}}{6} \\
& \gamma=-\bar{c}_{3} \bar{H}^{4} \bar{x}^{2}+\frac{\bar{c}_{2} \bar{H}^{2} \bar{x}}{3} \\
& \sigma=2 \bar{H}+2 \bar{c}_{3} \bar{H}^{3} \bar{x}^{3} \\
& \lambda=3 \bar{H}^{2}-2 \bar{c}_{3} \bar{H}^{4} \bar{x}^{3}+\frac{\bar{c}_{2} \bar{H}^{2} \bar{x}^{2}}{2}+\frac{\Omega_{r 0}}{a^{4}} \\
& \omega=2 \bar{c}_{3} \bar{H}^{4} \bar{x}^{2} .
\end{aligned}
$$

It is important to notice that the intrinsic values of the $c_{i}$ coefficients cannot be probed cosmologically. Only the various combinations of the $c_{i}$ 's and $x_{0}$ are relevant. The Friedmann equation which governs the evolution of the Hubble rate can be written in a similar way

$$
\bar{H}^{2}=\frac{\Omega_{m 0}}{a^{3}}+\frac{\Omega_{r 0}}{a^{4}}+\frac{\bar{c}_{2} \bar{H}^{2} \bar{x}^{2}}{6}-2 \bar{c}_{3} \bar{H}^{4} \bar{x}^{3}
$$

where the scalar energy density is $\frac{\rho_{\phi}}{H_{0}^{2} m_{\mathrm{Pl}}^{2}}=\frac{\bar{c}_{2} \bar{H}^{2} \bar{x}^{2}}{2}-6 \bar{c}_{3} \bar{H}^{4} \bar{x}^{3}$ and the scalar pressure $\frac{p_{\phi}}{H_{0}^{2} m_{\mathrm{Pl}}^{2}}=\frac{\bar{c}_{2}}{2} \bar{H}^{2} \bar{x}^{2}+$ $2 c_{3} \bar{H}^{3} \bar{x}^{2}(\bar{H} \bar{x})^{\prime}$ from which we define the equation of state $\omega_{\phi}=\frac{p_{\phi}}{\rho_{\phi}}$. The Friedmann equation gives the constraint on the parameters $1=\Omega_{m 0}+\Omega_{r 0}+\frac{\bar{c}_{2}}{6}-2 \bar{c}_{3}$ which determines $\bar{c}_{3}$ as a function of $\bar{c}_{2}$. 


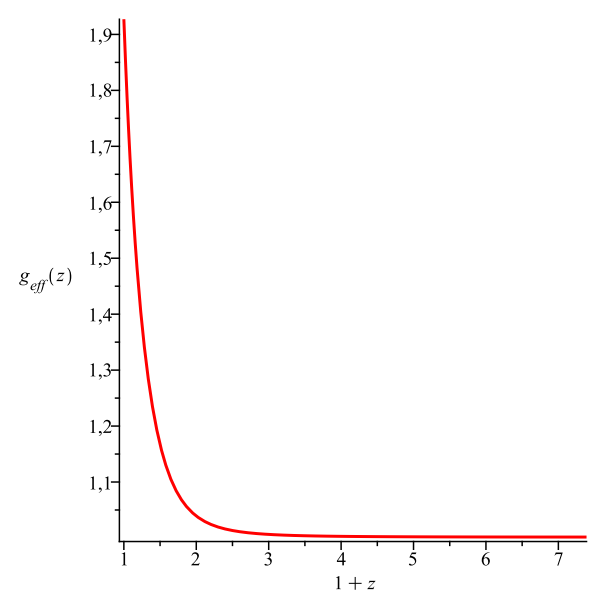

Figure 3: The rescaled Newton constant $g_{e f f}(z)$ for the cubic Galileon model with $\bar{c}_{2}=-4.3$.

The cubic Galileon models admit a long time attractor where both $\bar{x}^{\prime}$ and $\bar{H}^{\prime}$ vanish. This leads to self-acceleration with no cosmological constant in the model. The variation of the equation of state as a function of the redshift is shown in Figure 2.

The Galileon models modify gravity and in particular the growth of structure is altered. Defining by $\delta$ the density contrast of Cold Dark Matter (CDM), the growth equation becomes

$$
\delta^{\prime \prime}+\left(2+\frac{\bar{H}^{\prime}}{\bar{H}}\right) \delta^{\prime}-\frac{3}{2} \Omega_{m} g_{e f f} \delta=0
$$

where we have introduce the effective Newton constant in the FRW background

$$
g_{e f f} \equiv \frac{G_{e f f}}{G_{N}}=\frac{4\left(\kappa_{3} \kappa_{6}-\kappa_{1}^{2}\right)}{\kappa_{5}\left(\kappa_{4} \kappa_{1}-\kappa_{5} \kappa_{3}\right)-\kappa_{4}\left(\kappa_{4} \kappa_{6}-\kappa_{5} \kappa_{1}\right)} .
$$

where the various $\kappa_{i}^{\prime}$ 's are defined by

$$
\begin{aligned}
& \kappa_{1}=0, \kappa_{2}=-\frac{\bar{c}_{2}}{2}+6 \bar{c}_{3} \bar{H}^{3} \bar{x}, \kappa_{3}=-1 \\
& \kappa_{4}=-2, \kappa_{5}=2 \bar{c}_{3} \bar{H}^{2} \bar{x}^{2}, \kappa_{6}=\frac{\bar{c}_{2}}{2}-2 \bar{c}_{3}\left(\bar{H}(\bar{H} \bar{x})^{\prime}+2 \bar{H}^{2} \bar{x}\right)
\end{aligned}
$$

It is convenient to introduce the growth factor $f=\delta^{\prime}$ which measures the growth of structure and its deviation from the pure Einstein-de Sitter case where $f \equiv 1$. Deviations of growth from $\Lambda$-CDM are sizeable when $g_{e f f}$ is very different from one as in Figure 3 .

The parameter space of Galileon models has been scanned and compared to data [19], showing a preference for models with $c_{2}<0$ as we have in Figures 2 and 3. Although the models are ghostfree in the FRW background which solves the Galileon equations, Minkowski space is not a stable background of these models [20].

\section{Conclusion}

We have described the screening mechanisms of modified gravity. We have considered chameleons theories such as $f(R)$ in the large curvature limit for which the background evolution mimics $\Lambda$ $\mathrm{CDM}$. These models do not address the dark energy issue as they require to include a cosmological 
constant in their potential in order to lead to the acceleration of the Universe. On the other hand, they lead to interesting features on the growth of structure such as a scale dependent growth factor in the linear regime and deviations on scales smaller than $1 \mathrm{Mpc}$. Galileons offer the possibility of self-generating the acceleration of the Universe with no cosmological constant. They modify the growth of structure in a scale-independent way, the price to pay being that this happens when Minkowski space is not a stable background of the theory.

\section{References}

[1] P. Astier and R. Pain, Comptes Rendus Physique 13 (2012) 521.

[2] E. J. Copeland, M. Sami and S. Tsujikawa, Int. J. Mod. Phys. D 151753 (2006).

[3] T. Clifton, P. G. Ferreira, A. Padilla and C. Skordis, Phys. Rept. 513 (2012) 1 [arXiv:1106.2476 [astro-ph.CO]].

[4] A. Joyce, B. Jain, J. Khoury and M. Trodden, arXiv:1407.0059 [astro-ph.CO].

[5] B. Bertotti, L. Iess and P. Tortora, Nature 425 (2003) 374.

[6] J. G. Williams, S. G. Turyshev and D. Boggs, Class. Quant. Grav. 29 (2012) 184004 [arXiv:1203.2150 [gr-qc]].

[7] J. Khoury and A. Weltman, Phys. Rev. D 69, 044026 (2004).

[8] J. Khoury and A. Weltman, Phys. Rev. Lett. 93 (2004) 171104.

[9] P. Brax, C. van de Bruck, A. -C. Davis, J. Khoury and A. Weltman, Phys. Rev. D 70 (2004) 123518.

[10] E. Babichev, C. Deffayet and R. Ziour, Int. J. Mod. Phys. D 18 (2009) 2147 [arXiv:0905.2943 [hep-th]].

[11] A. I. Vainshtein, Phys. Lett. B 39 (1972) 393.

[12] W. Hu and I. Sawicki, Phys. Rev. D 76 (2007) 064004 [arXiv:0705.1158 [astro-ph]].

[13] C. Deffayet, G. Esposito-Farese and A. Vikman, Phys. Rev. D 79 (2009) 084003 [arXiv:0901.1314 [hep-th]].

[14] P. Brax and P. Valageas, Phys. Rev. D 90 (2014) 2, 023507 [arXiv:1403.5420 [astro-ph.CO]].

[15] P. Brax, A. C. Davis, B. Li and H. A. Winther, Phys. Rev. D 86 (2012) 044015 [arXiv:1203.4812 [astro-ph.CO]].

[16] P. Brax, A. -C. Davis and B. Li, Phys. Lett. B 715 (2012) 38 [arXiv:1111.6613 [astro-ph.CO]].

[17] L. Lombriser, Annalen Phys. 526 (2014) 259 [arXiv:1403.4268 [astro-ph.CO]].

[18] S. Appleby and E. V. Linder, JCAP 1203 (2012) 043 [arXiv:1112.1981 [astro-ph.CO]].

[19] J. Neveu, V. Ruhlmann-Kleider, A. Conley, N. Palanque-Delabrouille, P. Astier, J. Guy and E. Babichev, Astron. Astrophys. 555 (2013) A53 [arXiv:1302.2786 [gr-qc]].

[20] P. Brax, C. Burrage, A. C. Davis and G. Gubitosi, arXiv:1411.7621 [astro-ph.CO]. 